\title{
Interação e continuidade entre a aquisição da linguagem e a aprendizagem da leitura e da escrita ${ }^{1}{ }^{2}$
}

Ana Teberosky*

Maria Josep Jarque**

\begin{abstract}
*Catedrática em Psicologia Evolutiva e da Educação da Universidade de Barcelona e colaboradora associada do Laboratório de Educação
\end{abstract}

E-mail: ateberosky@ub.edu

**Professora do Departamento de Psicologia Evolutiva e de Educação da Universidade de Barcelona

E-mail:mj_jarque@ub.edu

\begin{abstract}
Resumo: Diversos estudos concordam que a leitura em voz alta para as crianças pequenas pelos adultos tem influência significativa na elevação dos níveis de conhecimento do vocabulário e, consequentemente, de sua competência linguística oral (Mol, Bus e de Jong, 2009, para uma metaanálise), indicando que a extensa preparação do adulto (pai ou professor) pode ser necessária se quisermos influenciar essa competência linguística. Neste artigo, discutimos em qual sentido o conhecimento do vocabulário e as estruturas linguísticas que o incluem contribuem para o desenvolvimento linguístico das crianças pequenas.
\end{abstract}

Palavras-chave: Desenvolvimento da linguagem. Vocabulário. Leitura em voz alta.

\footnotetext{
Este estudo faz parte de um amplo projeto, inserido no contexto de um convênio com o Laboratório de Educação (http://www.labedu.org.br/) do Brasil, no qual também participam Núria Ribera e Júlia Coromina.

2 Tradução de Evandro Lisboa Freire; revisão técnica de Angélica Sepúlveda.
} 
A aquisição da língua para as crianças pequenas e a aprendizagem da leitura e da escrita para os estudantes fazem parte do processo de conhecimento da linguagem que, como afirma Goldberg (1995), trata-se de um processo de conhecimento. O conhecimento não é um processo unitário, porque há uma variedade de conhecimentos: factual ou perceptivo, habilidades motoras ou sentimentos, etc., mas o conhecimento da linguagem não é apenas mais um na classificação acima. Com sua aquisição, as crianças começam a ter acesso a um sistema simbólico de significados, a um recurso por meio do qual a experiência pode transformar-se em conhecimento. A relação entre a linguagem e a aprendizagem é baseada na interação entre a aquisição e o uso da língua na construção do conhecimento por meio do falar e do pensar.

As declarações acima correspondem a uma perspectiva para a qual as estruturas da linguagem surgem a partir de seu uso (BYBEE, 2010; CROFT, 2001; GOLDBERG, 1995; LANGACKER, 1987, 1991; TOMASELLO, 2003). Com esse uso da língua, as crianças também estão aprendendo por meio desta e os novos modos de linguagem são novas formas de conhecimento. Sob essa perspectiva, a linguagem é a base da aprendizagem e a competência linguística madura é concebida como um inventário estruturado de estruturas linguísticas significativas.

Como em qualquer outro processo de aprendizagem de atividades cognitivas complexas, os aprendizes linguísticosaprendem como materialverbal oferecido a eles, isto é, o input (entrada), e com ele constroem as categorias abstratas e os esquemas imitando as coisas concretas que ouvem (TOMASELLO, 2003). Por isso, a contribuição do adulto que interage e comunica-se com a criança não é considerada uma variável externa, mas um componente interno muito importante no processo de estruturação da linguagem. Em que sentido o adulto contribui com o desenvolvimento da linguagem? Trata-se de uma parte do processo porque, por meio de uma conduta comunicativa, o adulto oferece modelos para imitação, correções, repetições, reformulações e ampliações dos enunciados das crianças. Esses modelos da interação dialógica inicial em um adulto informado são estendidos, então, em modelos de leitura por meio da leitura em voz alta de contos e poemas voltados às crianças e dos comentários sobre o que foi lido. Esse tipo de aulas específicas de língua oral e escrita em um adulto informado foi identificado como elemento facilitador do desenvolvimento da linguagem, não só em nível geral, mas, também, em aspectos específicos, como a aprendizagem do vocabulário, o que, por sua vez, não só aumenta o conhecimento lexical, mas ajuda no desenvolvimento fonológico, morfológico, sintático e discursivo. 
Esse aspecto de base lexical da linguagem levou a linguística cognitiva a propor que a gramática emerge do léxico e do uso na experiência, como nos sistemas complexos que foram identificados, por exemplo, na biologia. De modo similar, na aquisição da linguagem, o léxico constitui uma das bases da aprendizagem (BATES; GOODMAN, 1997; TOMASELLO, 2003). Os fatores relacionados ao léxico são a frequência no input, a frequência dos exemplares e a diversidade dos tipos de estruturação. Nesse sentido, maior frequência e diversidade se encontram na modalidade escrita e em todas as formas de linguagem letrada. Assim, qual é a forma de difusão da linguagem escrita para as crianças de 4 e 5 anos que ainda não são leitoras independentes? 0 melhor procedimento de difusão é a leitura em voz alta, como ocorreu ao longo da história cultural da humanidade durante séculos entre a população não escolarizada (FRENK, 2004).

Vejamos primeiro alguns aspectos desse processo interativo entre léxico e fonologia, morfologia, sintaxe e discurso e, em seguida, abordemos a continuidade entre as modalidades oral e escrita.

\section{FONOLOGIA E LÉXICO}

A maioria das investigações situa em torno de 4 a 5 anos o fechamento de uma primeira etapa da aquisição do sistema fonológico (BOSCH, 2004; INGRAM, 1983). Posteriormente, segue-se um período de aperfeiçoamento que pode estender-se até os 6 ou 7 anos, inclusive de alguns aspectos fonéticos como a sonoridade ou o local de articulação das oclusivas - em relação aos quais os alunos não desenvolverão habilidades similares às dos adultos até a adolescência (LLACH; PALMADA, 2011).

A pesquisa acerca da aquisição da linguagem tem mostrado uma relação direta entre o desenvolvimento do léxico e o desenvolvimento fonológico. Nesse sentido, Ferguson e Farwell (1975) foram alguns dos primeiros a destacar que um núcleo fônico de itens lexicais na memória e nas articulações que os produzem constitui a base para a fonologia no falante. Ou seja, os aprendizes precisam dispor de uma "massa crítica" de léxico suficiente para poder abstrair os segmentos e as estruturas fonológicas a partir das palavras que compõem seu vocabulário.

No processo de desenvolvimento da linguagem, observamos diferentes fenômenos que expressam a interação que ocorre entre a fonologia e o léxico. Especificamente, as pesquisas reunidas em Storkel e Morrisette (2002) 
demonstraram efeitos da frequência lexical, da densidade da vizinhança e da probabilidade fonotática.

Por um lado, o conhecimento lexical influencia a aquisição fonológica. Diversos estudos indicam que crianças que dispõem de um léxico completo tendem a produzir maior variedade de sons, assim como de combinações de sons, enquanto crianças que possuem um conhecimento lexical insuficiente tendem a produzir menor variedade de sons, bem como combinações limitadas entre eles (STORKEL; MORRISETTE, 2002). Por outro lado, a frequência lexical foi observada em relação aos processos de produção e percepção. As palavras com maior frequência tendem a ser produzidas com maior correção que aquelas com baixa frequência.

Quanto aos efeitos relacionados ao tipo de vizinhança, estes estão ligados à organização das palavras. O vocabulário, tanto do adulto como do aprendiz, é organizado por meio de conexões entre as palavras de diversos tipos (semânticas, fonológicas, de uso, etc.). As estruturas resultantes da similaridade fonética são denominadas vizinhança. 0 número de vizinhanças em que determinada palavra participa de modo concreto varia de acordo com a condição de o falante conhecer muitas ou poucas palavras formalmente parecidas, resultando em vizinhanças densas e vizinhanças reduzidas.

Entretanto, o processo interativo não termina com a "massa crítica" do léxico, também encontramos relação entre estruturas oracionais e aspectos fonológicos, especialmente com a prosódia.

\section{FONOLOGIA E ESTRUTURAS: PROSÓDIA}

As unidades prosódicas constituem um componente essencial das estruturas sintáticas e discursivas. Assim, por exemplo, as diferenças da modalidade oracional (oração declarativa, interrogativa ou imperativa) são expressas em algumas línguas apenas por uma mudança do contorno de entonação, como em português ou em catalão, ou pela combinação de um contorno de entonação e outros mecanismos linguísticos, tais como alterar a ordem dos elementos, pronomes interrogativos, etc. Por outro lado, a prosódia também é relevante na resolução de ambiguidades sintáticas, como para a atribuição de papéis temáticos em línguas nas quais a ordem dos elementos pode variar e não se dispõe de marcação de caso ou onde esta é ambígua. A marcação prosódica também é fundamental para localizar limites entre os elementos (BOLINGER, 1985). A partir dos 4 anos, o sistema de entonação torna-se mais 
complexo e prossegue a aquisição de diferentes aspectos relacionados à compreensão e produção da entonação. Wells et al. (2004) ilustram como a aquisição da entonação se estende além dos 6 anos.

O processo de aprendizagem continua a ser interativo, entre a frequência de uso lexical e o desenvolvimento semântico.

\section{SEMÂNTICA E LÉXICO}

Foi descrito que até os 2 anos ocorre uma explosão de vocabulário: as crianças aprendem muitas palavras novas por dia, até chegar a um vocabulário de produção entre 700 e mil palavras aos 3 anos de idade (FENSON et al., 1994). Entre os 3 e 4 anos continua a aumentar o vocabulário em número de palavras com conteúdo lexical e de palavras funcionais, mas a quantidade não constitui a única mudança.

Até os 3 anos, há uma primeira reorganização do vocabulário em relação aos significados dos itens lexicais que se agrupam por compartilhar aspectos comuns. Os significados compartilhados por grupos estão relacionados aos domínios conceituais, ou seja, os significados se encontram em inter-relação com uma modelagem do conhecimento, ligada, por sua vez, à experiência dos falantes (conhecimento do tipo enciclopédico), assim como às formas convencionais da língua que as crianças ouvem.

Quando as crianças adquirem expressões, adquirem o conhecimento associado a elas. Ao nomear e comentar a experiência, as crianças estão aprendendo conceitos e categorizando esse conhecimento. O rápido crescimento do vocabulário das crianças aumenta as relações de significação e tem um desenvolvimento paralelo da categorização (PAINTER, 1999). As relações de significação das palavras coexistem na designação de um mesmo referente, com características distintas ou a partir de perspectivas distintas. Esse é o caso das relações semânticas entre os termos, relações de hiperonímia, sinonímia, antonímia e meronímia. Diversos autores mostram que entre os 2 e 3 anos, as crianças aceitam que palavras diferentes designem o mesmo referente, mas de maneiras distintas. Por exemplo, os nomes próprios e nomes básicos podem servir para nomear um animal de estimação, uma mesma imagem pode ser denominada gato e animal, uma nova palavra pode referirse a uma parte do objeto, a um aspecto diferente, a uma nova informação sob uma perspectiva diferente, etc. (TOMASELLO, 2003). Portanto, a hipótese da rede é relevante principalmente quando aplicada ao vocabulário. Uma 
palavra constitui o núcleo de uma rede de homônimos, sinônimos, classes de palavras e de colocações, cada uma das quais é, por sua vez, o centro de outra pequena rede.

A partir dos 4 ou 5 anos e com a escolaridade e a aprendizagem da escrita, há outra explosão de vocabulário, com a qual se dispõem de 20 mil a 50 mil palavras em produção, tornando-se ainda maior a compreensão. A leitura de textos ajuda a ampliar a rede de relações entre palavras, relações não só sintagmáticas, mas, também, paradigmáticas. Quando uma criança aprende um novo item de vocabulário, adiciona uma pequena rede com vínculos com outros nodos, incluindo sua pronúncia, seu significado, etc. Uma palavra (ou qualquer outro conceito) que possui apenas um par de vínculos mal é integrada e logo é esquecida, porém, uma rica coleção de vínculos garante à palavra uma vida longa e útil (HUDSON, 2003).

O léxico também interage com o desenvolvimento morfológico, nesse caso, os fatores de frequência se relacionam com a frequência de exemplares mais ou menos fixos.

\section{MORFOLOGIA E LÉXICO}

As palavras que constituem o léxico possuem diferentes graus de fixação. Isso se deve, fundamentalmente, à frequência de uso de cada palavra ou à frequência do exemplar. As palavras com alto grau de fixação são mais facilmente acessíveis, servem de base nas relações morfológicas e têm uma autonomia que as torna mais resistentes à mudança, perdem sua transparência semântica e sintática e criam uma forte representação (BYBEE, 1995). As palavras se relacionam com outras por meio do conjunto de conexões entre idênticos ou semelhantes traços fonológicos e semânticos. Por exemplo, em espanhol, em francês e em outras línguas românicas, os verbos são distribuídos em várias classes de conjugação, sendo que o maior número de tipos pertence à primeira conjugação; as outras conjugações tendem a ter poucos verbos, que são tipicamente altos em termos de frequência de exemplares. A frequência de exemplar, em vez de tipo, é aprendida de memória se houver quantidade suficiente de input (BYBEE, 1995). 
Se o léxico interage com o desenvolvimento morfológico, também o faz com a morfossintaxe.

\section{MORFOSSINTAXE E ESTRUTURAS FIXAS}

Uma das implicações do fato de que o uso da linguagem é determinado lexicalmente é que certas palavras tendem a ser utilizadas em certas colocações ou estruturas (BYBEE, 2010). Se considerarmos, ainda, as estruturas perifrásticas verbais, os emparelhamentos entre verbos e preposições (estar interessado em, pensar que, pensar sobre), o total de estruturas pré-fabricadas é muito alto. Essas colocações convencionais tornam a ser repetidas no discurso. Erman e Warren (2000) constataram que constituem cerca de $55 \%$ das produções, tanto na modalidade falada como na escrita. Os locutores reconhecem as estruturas pré-fabricadas como familiares, indicando que essas sequências de palavras são armazenadas na memória, apesar de ser bastante previsíveis em termos de forma e significado (BYBEE, 2010).

E o léxico também é fundamental para as funções textuais de coerência e coesão.

Texto, discurso e LÉXICO

O léxico tem diversas funções no discurso: especificar a referência e orientar todas as relações entre os elementos referenciais do discurso - relações anafóricas, de especificação ou de descrição. Por exemplo, as relações entre substantivos, sintagmas nominais, pronomes pessoais e possessivos que se referem à pessoa, ou as relações de designação dos artigos definidos ou indefinidos ou as relações semânticas do léxico de hiperonímia, antonímia ou sinonímia. São esses os elementos que carregam o peso da referência no discurso: é por meio das palavras, das marcas morfológicas, da ordem das palavras, das relações entre elas, além da prosódia, que o falante pode identificar do que se está falando.

$\mathrm{Na}$ interação face a face, a referência é aprendida com palavras que foram denominadas “vocabulário básico", ou seja, palavras que designam entidades concretas e palavras relacionais, como os verbos ou as preposições. Em contrapartida, o "vocabulário não básico" e as palavras com funções anafóricas não encontram suporte extralinguístico nem designam entidades diretamente observáveis (DABROWSKA, 2009). Eles são construídos no uso 
discursivo, quando se aprende a utilizar nomes comuns ou próprios, sintagmas nominais, substantivos e determinantes, pronomes ou referência nula de acordo com o contexto. Trata-se de relações semânticas e de coerência que depois adotarão funções textuais, assim como os conectores e as expressões que fixam e estruturam o texto.

\section{CONCLUSÕES}

Em conclusão, qual é a interação entre o conhecimento infantil da língua falada e a aprendizagem da língua escrita? Muitas palavras e estruturas são utilizadas em ambas as modalidades, oral e escrita, embora haja diferenças devido ao fato de que a fala é produzida em tempo real, enquanto no modo escrito há uma cuidadosa revisão e edição (BIBER, 2009). Por exemplo, Hayes e Ahrens (1988) analisam a frequência e a complexidade das palavras em diferentes contextos e apontam que os alunos mais velhos encontramse expostos a palavras novas principalmente em textos escritos: nos livros há uma quantidade $50 \%$ maior de palavras raras que na televisão ou nas conversas entre adultos educados.

Se o léxico e as estruturas em seu entorno têm tanta importância para o desenvolvimento da linguagem, podemos concluir que se a aprendizagem da linguagem oral ocorrer em contextos de leitura em voz alta, de interação com livros e leitores, ou seja, contextos letrados, incluirá as formas escritas e, com elas, sua frequência, sua fixação, seus contextos e formas de estruturação da linguagem. 


\section{Interaction and continuity between language acquisition and the learning of reading and writing}

Abstract: Several studies agree that reading aloud for young children has a significant impact in raising children's levels of knowledge and vocabulary and, consequently, their oral linguistic competence (Mol, Bus and de Jong, 2009 for a meta-analysis), indicating that the extensive preparation of the adult (parent or teacher) can be necessary if we are to influence this linguistic competence. In this article, we discuss in what sense the knowledge of vocabulary and the linguistic structures that include it can contribute to the linguistic development of young children.

Keywords: Language development. Vocabulary. Reading aloud. 


\section{REFERÊNCIAS}

BATES, E.; GOODMAN, J. On the inseparability of grammar and the lexicon: evidence from acquisition, aphasia and real-time. Language and Cognitive Processes, v. 12, n. 5/6, p. 507-584, 1997

BIBER, D. Are there linguistic consequences of literacy? Comparing the potentials of language use in speech and writing. In: OLSON, D. R.; TORRANCE, N. (Ed.). Cambridge Handbook of Literacy. Cambridge: Cambridge University Press, 2009, p. 75-91.

BOSCH, L. Evaluación fonológica del habla infantil. Barcelona: Masson, 2004.

BYBEE, J. L. Regular morphology and the lexicon. Language and Cognitive Processes, v. 10, n. 5, p. 425-255, 1995.

Language, usage and cognition. Cambridge: Cambridge University Press, 2010

BOLINGER, D. L. Intonation and its parts. Londres: Edward Arnold, 1985.

CROFT, W. Radical construction grammar: syntatic theory in typological perspective. Oxford: Oxford University Press, 2001.

DABROWSKA, E. Words as constructions. In: EVANS, V.; POURCEL, S. (Ed.). New directions in cognitve linguistics. Amsterdã: Johns Benjamins, 2009, p. 201-223.

ERMAN, B.; WARREN, B. The idiom principle and the open choice principle. Text, v. 20, n. 1, p. 29-62, 2000.

FENSON, L. et al. Variability in early communicative development. Monographs of the Society for Research in Child Development, 59 (Serial number 242), 1994.

FERGUSON, C.; FARWELL, C. Words and sounds in early language acquisition. Language, v. 51, n. 2, p. 419-429, 1975.

FRENK, M. Oralidad y escritura y lectura. In: RICO, F. (Ed.). Don Quijote de la Mancha. Madri e México: Real Academia Espanola, Asociación de Academias de la Lengua Espanola, Editorial Alfaguara, 2004, p. 1138-1144.

GOLDBERG, A. E. Constructions: a construction grammar approach to argument structure. Chicago: Chicago University Press, 1995. 
HAYES, D. P.; AHRENS, M. Vocabulary simplification for children: a special case of ,motherese‘? Journal of Child Language, v. 15, p. 395-410, 1988.

HUDSON, R. Word grammar. In: FRAWLEY, W. (Ed.). International Encyclopedia of Linguistic. 2.ed. Oxford: Oxford University Press, 2003.

INGRAM, D. Trastornos fonológicos en el nino. Barcelona: Médica y Técnica, 1983.

LANGACKER, R. W. Foundations of cognitive grammar. Stanford: Stanford University Press, 1987.

. Foundations of Cognitive Grammar. Standford: Stanford University Press, 1991. (v. 2. Descriptive Application.)

LLACH, S.; PALMADA, B. Cambios en la adquisición del sistema fonológico de las consonantes entre 4 y 5 anos. Revista de Logopedia, Foniatría y Audiología, v. 31, n. 2, p. 106-112, 2011.

MOL, S.; BUS, A.; DE JONG, M. Interactive book reading in early education: a tool to stimulate print knowledge as well as oral language. Review of Educational Research, v. 79, n. 2, p. 979-1007, 2009.

PAINTER, C. Learning through language in early childhood. Londres: Continuum, 1999.

STORKEL, H. L.; MORRISETTE, M. L. (2002). The lexicon and phonology: interactions in language acquisition. Language, Speech, and Hearing Services in Schools, n. 33, p. 24-37, 2002.

TOMASELLO, M. (2003). Constructing a language: a usage-based approach to child language acquisition. Cambridge, MA: Harvard University Press, 2003.

WELLS, B.; PEPPÉ, S.; GOLANDRIS, N. Intonation development from five to thirteen. Journal of Child Language, n. 31, p. 749-778, 2004.

RECEBIDO: Maio de 2014

APROVADO: Junho de 2014 